\title{
Contribution to the damage modelling of reinforced concrete structures
}

\section{Contribution à la modélisation de l'endommagement des structures en béton armé}

\author{
Mourad KHEBIZI ${ }^{1}$, Hamza GUENFOUD ${ }^{2,}$ Mohamed GUENNFOUD ${ }^{2}$ \\ ${ }^{1}$ Université des frères Mentouri, Constantine, Algérie, E-mail : Mourad_gc@yahoo.fr \\ ${ }^{2}$ Laboratoire de génie civil et d'hydraulique, Université de Guelma, Algérie
}

\begin{abstract}
A two-dimensional multi-layered finite elements modeling of reinforced concrete structures at nonlinear behaviour under monotonic and cyclical loading is presented. The non-linearity material is characterized by several phenomena such as: the physical non-linearity of the concrete and steels materials, the behaviour of cracked concrete and the interaction effect between materials represented by the post-cracking field. These parameters are taken into consideration in this paper to examine the response of the reinforced concrete structures at the non-linear behaviour. Two examples of application are presented. The numerical results obtained, are in a very good agreement with available experimental data and other numerical models of the literature.
\end{abstract}

\begin{abstract}
Résumé. Une Modélisation bidimensionnelle par éléments finis multicouches du comportement endommageant des structures en béton armé sous charge monotone et cyclique est présentée. La non linéarité matérielle est caractérisée par plusieurs phénomène tels que : la non-linéarité physique des matériaux béton et acier, le comportement du béton fissuré, l'effet d'interaction entre les matériaux représentée par le domaine post-fissuration. Ces paramètres sont pris en considération dans cet article pour examiner la réponse des structures en béton armé à comportement non linéaire. Deux exemples d'applications sont présentés. Les résultats numériques obtenus, sont en concordance très favorable avec ceux obtenus par l'expérience et d'autres modèles numériques de la littérature.
\end{abstract}

\section{Introduction}

La réponse d'une structure sous un chargement résulte d'une forte interaction entre les effets des matériaux (non-linéarités locales), les effets de géométriques des structures (géométrie, répartition des forces et des raideurs, liaisons) et l'effet d'environnement (interaction sol-structure). Les non-linéarités locales sont notamment liées à la formation, à l'ouverture et la refermeture des fissures d'une part, à la liaison et au comportement des armatures (plastification des aciers) d'autre part. Une bonne description de ces phénomènes est un passage obligé si l'on veut représenter les variations des raideurs de la structure et suivre le comportement jusqu'à la ruine.

Dans cet article nous présentons une méthode de modélisation numérique des structures planes (2D) en béton armé sous chargement statique et cyclique, elle utilise des éléments poutres de type multicouches dont la matrice de raideur est calculée à l'aide d'une discrétisation de la poutre selon la hauteur en couches successives superposées (figure 1). La sommation de ces couches permet le calcul de la raideur d'une manière correcte et la prise en compte des variations du comportement. L'hypothèse cinématique de Bernoulli confère aux différentes couches un comportement uniaxial [1]. Ceci permet de traiter les comportements locaux à travers des lois uniaxiales, pour le béton et l'acier, attribuées à chaque couche. Le calcul des efforts anélastiques s'effectue grâce à une méthode d'itération basée sur la raideur sécante initiale [3]. Un traitement particulier est réservé aux couches comprenant simultanément du béton et d'acier. Le comportement des couches mixtes est homogénéisé par la loi de mélange permettant ainsi de calculer la contrainte de la couche au prorata de chaque matériau [4-6].

$\sigma_{\text {couche }}=(1-A) \sigma_{\text {béton }}+A \sigma_{\text {acier }}$ 
Tel que $\sigma_{\text {couche }}$ représente la contrainte axiale de la couche mixte, $\sigma_{\text {béton }}$ : contrainte du béton dans la couche mixte, $\sigma_{\text {acier }}$ : contrainte d'acier dans la couche mixte et $A$ : représente l'aire relative d'acier au sein de la couche renforcée.

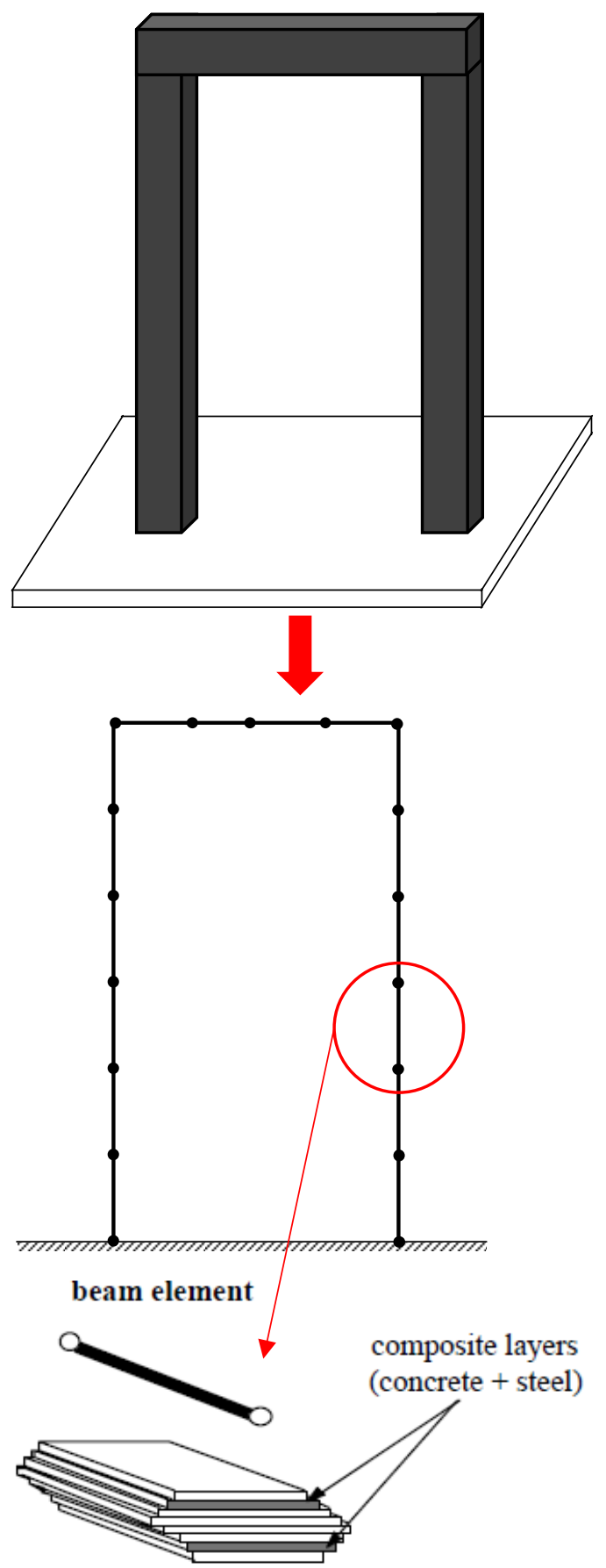

Fig. 1. Principe de discrétisation des structures en béton armé par des éléments poutres multicouches.

\section{Modèle d'endommagement pour le béton}

Le comportement du béton est décrit dans cet article par le modèle unilatéral de Laborderie [7], il est adapté à la description du comportement endommageable généré par la création de microfissures (abaissement des raideurs) et le fonctionnement lié, au cours de cycles, à leur refermeture (unilatéralité). Deux variables d'endommagement scalaire sont utilisées, l'une en traction $\mathrm{D}_{1}$, l'autre en compression $\mathrm{D}_{2}$, agissant sur la rigidité des matériaux et générant des déformations permanentes. La réponse uniaxiale du modèle soumis à un cycle de traction-compression est présentée à la figure 2.

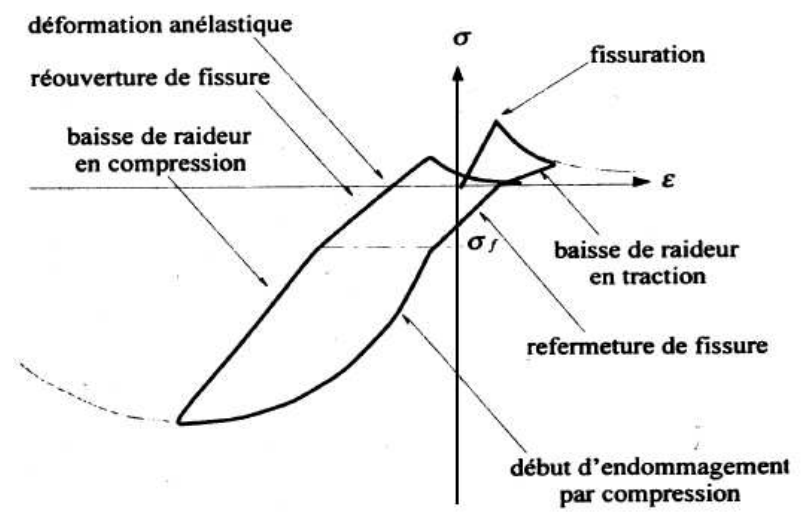

Fig. 2. Réponse uniaxiale du modèle Unilatéral [7]

\section{Applications}

\subsection{Modélisation d'une poutre en béton armé}

Dans cette section nous utilisons des éléments finis multicouches pour modéliser la poutre MECA [9]. La géométrie et les conditions de chargement sont présentées dans la figure $3 . \mathrm{Vu}$ son élancement, cette structure se comporte comme une poutre dominée par la flexion. L'influence du cisaillement est négligeable et une modélisation de type poutre multicouche de Bernoulli, avec lois de comportement uniaxial pour le béton et l'acier, est suffisante [1]. Le comportement du béton suit le modèle d'endommagement de Laborderie [LAB 91], les caractéristiques mécaniques considérées pour le béton et l'acier sont présentées dans le tableau 1. Dans ce travail nous avons modélisé la structure par 10 éléments poutres à 2 nœuds avec 2 points d'intégration, la section de chaque élément est discrétisée par 10 couches superposées, dont 8 en béton seul et 2 comprenant simultanément du béton et de l'acier (figure 4). Ragueneau [3] a étudié numériquement (3D) la réponse de la même poutre.
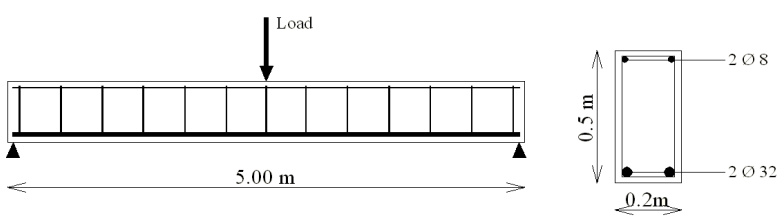

Fig. 3. Poutre en béton armé du MECA benchmark. 
Table 1. Caractéristiques utilisées pour le modèle de comportement de Laborderie [5].

\begin{tabular}{|c|c|}
\hline Paramètre & valeur \\
\hline Module de Young & $37272 \mathrm{e}^{6} \mathrm{~Pa}$ \\
\hline Masse volumique & $2400 \mathrm{~kg} / \mathrm{m} 3$ \\
\hline $\begin{array}{c}\text { Seuil d'endommagement en traction } \\
\text { compression }\end{array}$ & $310 \mathrm{~Pa}$ \\
\hline $\begin{array}{c}\text { Seuil d'endommagement en } \\
\text { traction }\end{array}$ & $7000 \mathrm{~Pa}$ \\
\hline $\begin{array}{c}\text { Paramètre d'endommagement en } \\
\text { compression }\end{array}$ & $9 \mathrm{e}^{-3} \mathrm{~Pa}-1$ \\
\hline $\begin{array}{c}\text { Paramètre d'endommagement en } \\
\text { Paramètre pour la traction }\end{array}$ & $5.30 \mathrm{e}^{-6} \mathrm{~Pa}-1$ \\
\hline $\begin{array}{c}\text { Paramètre pour la compression } \\
\text { permanente en traction }\end{array}$ & 1.20 \\
\hline $\begin{array}{c}\text { Activation de la déformation } \\
\text { permanente en compression }\end{array}$ & $-40 \mathrm{e}^{6} \mathrm{~Pa}$ \\
\hline $\begin{array}{c}\text { Contrainte de refermeture des fissures } \\
\text { con de la déformation }\end{array}$ & $3.50 \mathrm{e}^{6} \mathrm{~Pa}$ \\
\hline
\end{tabular}

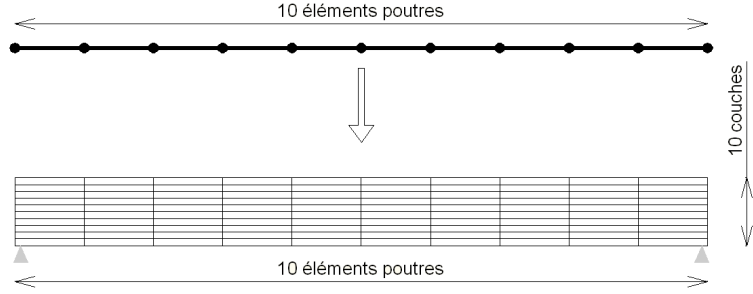

Fig. 4. Discrétisation en éléments multicouches.

La figure 6 représente une comparaison entre les résultats obtenus par notre simulation numérique, ceux de Ragueneau [3] et les résultats expérimentaux extraits obtenus lors du benchmark MECA [9]. Nous voyons que notre modélisation donne dans l'ensemble des résultats comparables avec ceux Ragueneau [3] d'une part et les résultats expérimentaux d'autre part. Cependant, les légères différences entre les résultats peuvent être imputables à un effet de maillage de la section de la poutre.

La figure 5 représente la carte d'endommagement de traction obtenue par la présente modélisation, cet endommagement est localisé au milieu de la fibre inférieure de la poutre, et se propage tout le long de celleci.

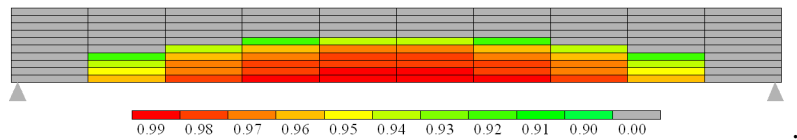

Fig. 5. Carte d'endommagement de traction «D1».

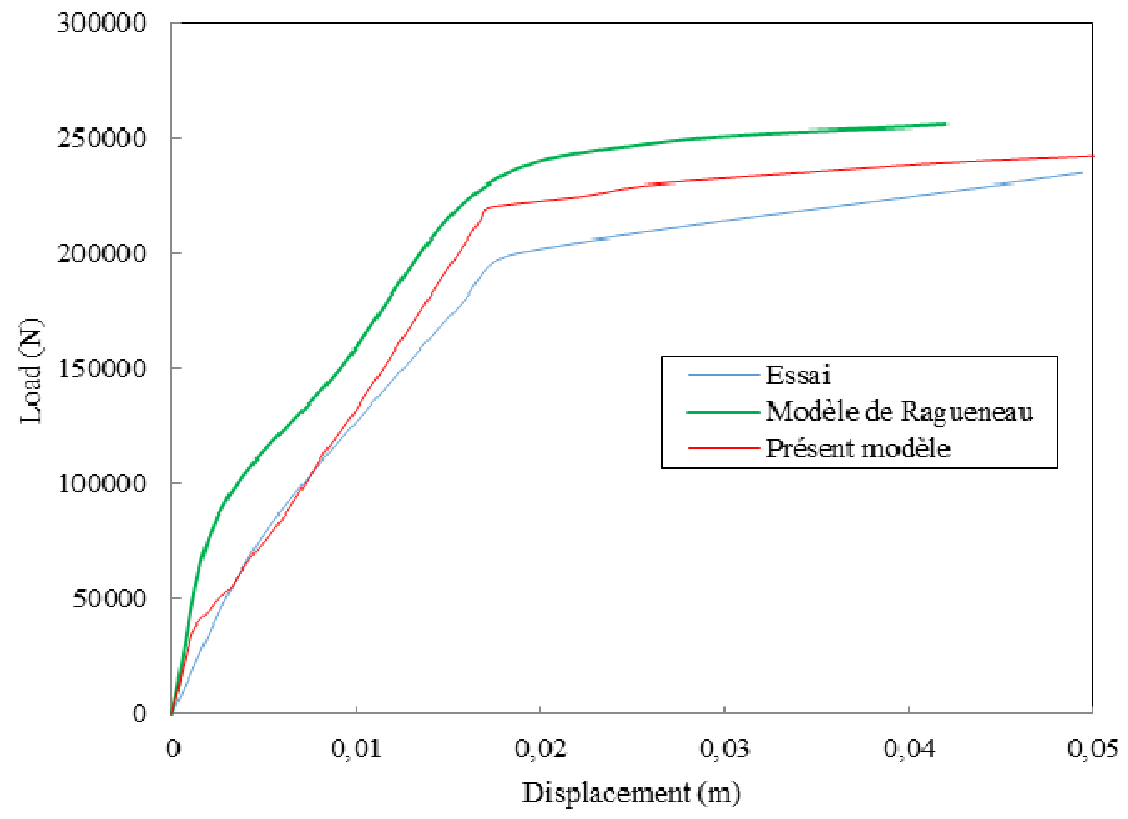

Fig. 6. Courbe charge-flèche de la poutre MECA. 


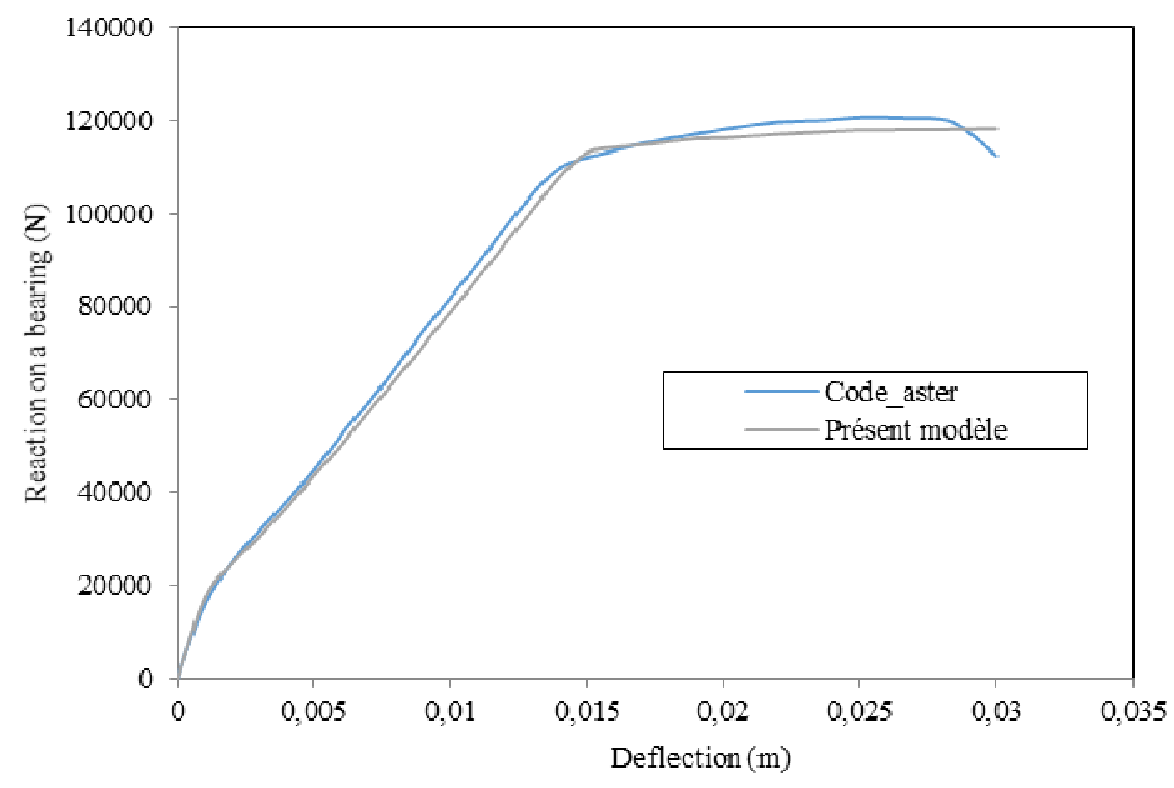

Fig. 7. Courbe réaction d'appui- flèche à mi- travée.

\subsection{Modélisation de la réponse cyclique d'une poutre en béton armé}

On va modéliser maintenant le comportement cyclique d'une poutre en béton armé dont la géométrie est présentée dans la figure 8 . Le chargement est composé d'un cycle d'amplitude $1 \mathrm{~mm}$ suivi d'un cycle d'amplitude $2 \mathrm{~mm}$ (figure 9).
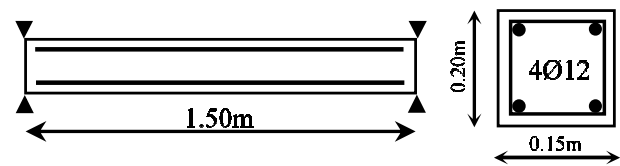

Fig. 8. Géométrie et armatures des poutres.

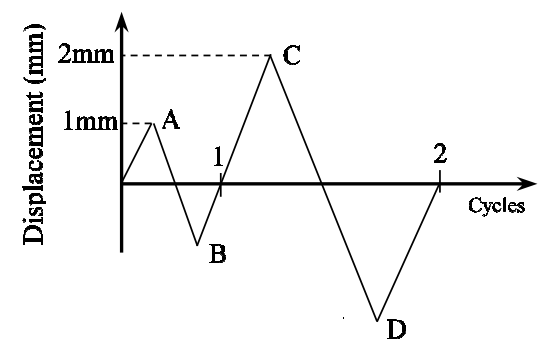

Fig. 9. Histoire de chargement.

La structure est modélisée dans ce travail par 20 éléments poutres à 2 nœuds et 2 points d'intégration, la section de chaque élément est discrétisée par 10 couches superposées, dont 8 en béton seul et 2 comprenant simultanément du béton et de l'acier. Le comportement du béton suit la loi d'endommagement de Laborderie [7], les caractéristiques mécaniques considérées pour le béton et l'acier sont présentées dans [5].

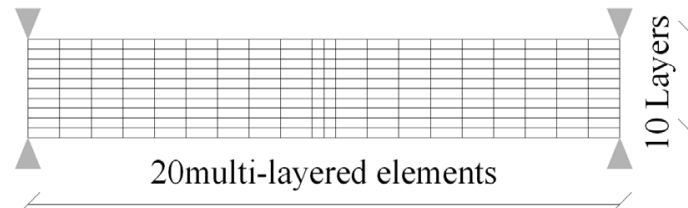

Fig. 10. Discrétisation de la poutre en éléments multicouches.

La figure 11 compare la réponse de la poutre décrite par notre modélisation et celle obtenue expérimentalement par Laborderie [7], on remarque une très bonne cohérence entre les deux réponses. La figure 12 présente également une comparaison de la réponse force-flèche décrite par notre simulation et celle de Matallah [10] obtenue par un modèle de comportement basé sur le couplage de l'endommagement et le l'anélasticité. Les deux modèles numériques donnent des résultats similaires dans le premier cycle du chargement. Cependant, pour le deuxième cycle de chargement, des légères différences sont observées. Les figures 12.a et 12.b présentent la carte d'endommagement de la poutre pour le premier cycle de chargement. A l'état de chargement « A » la partie supérieure de la poutre est endommagée (figure 12.a). L'état de chargement « $\mathrm{B} »$ correspond à un chargement opposé, l'état d'endommagement initialement produit est toujours conservé en mémoire alors qu'un nouveau état d'endommagement se crée dans la partie inférieure de la poutre (figure 12.b). Les figures 13.a et 13.b présentent la carte d'endommagement de la poutre au cours du deuxième cycle de chargement. 


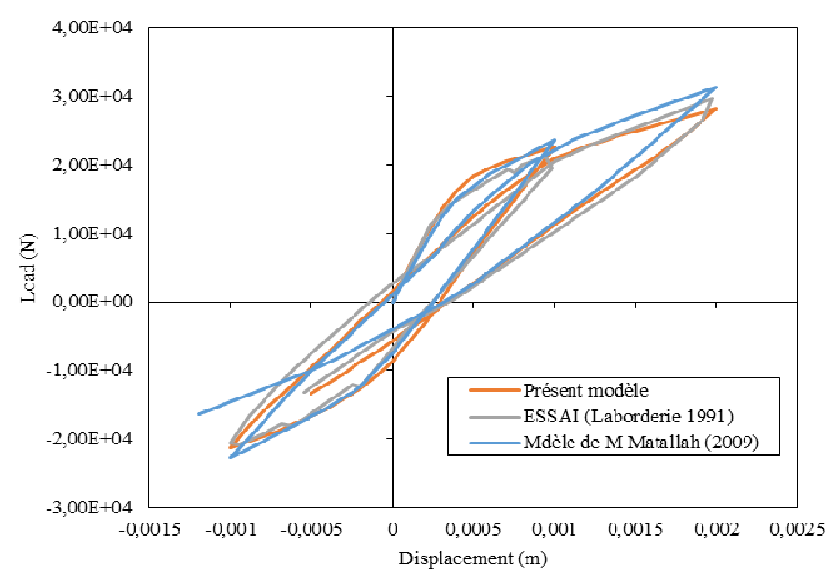

Fig. 11. Comparaison de la réponse force-flèche entre la simulation numérique et l'expérience.

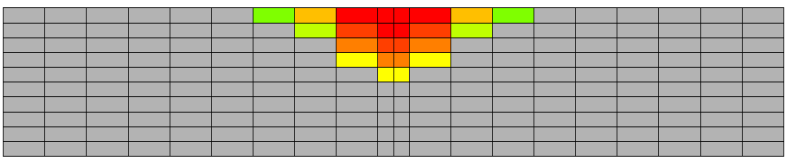

$\begin{array}{lllllllll}0.90 & 0.80 & 0.70 & 0.60 & 0.50 & 0.40 & 0.30 & 0.00\end{array}$

(a) Etat de chargement $\mathrm{A}$

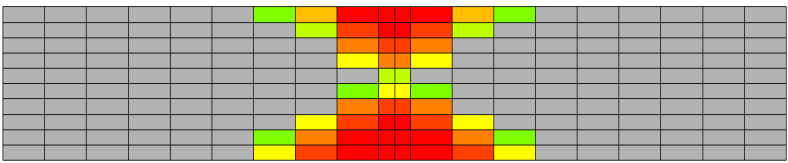

\begin{tabular}{lllllllllll}
\hline 0.90 & 0.80 & 0.70 & 0.60 & 0.50 & 0.40 & 030 & 0.00
\end{tabular}

(b) Etat de chargement B

Fig. 12. Carte d'endommagement de traction «D1» pour le premier cycle de chargement.

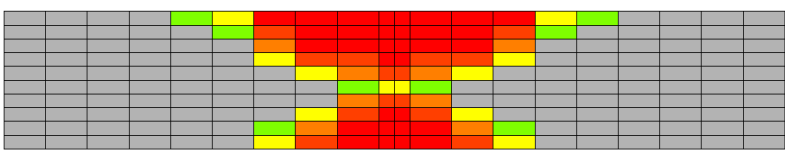

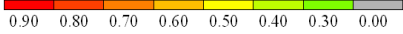

(c) Etat de chargement $\mathrm{C}$

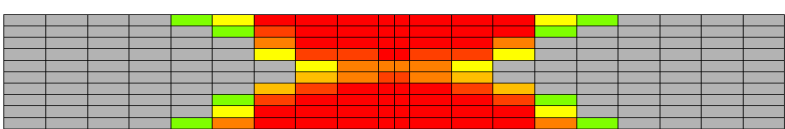

(d) Etat de chargement D

Fig. 13. Carte d'endommagement de traction $« \mathrm{D} 1 »$ pour le deuxième cycle de chargement.

\section{Conclusion}

Une modélisation simple du comportement non linéaire des structures en béton armé est présentée. Elle utilise des éléments poutres multicouches obéissant à l'hypothèse de Bernoulli pour conférer aux différentes couches un comportement uniaxial. Elle permet également la description de l'état d'endommagement des structures au cours d'un chargement. Deux exemples d'applications ont été présentés. Le premier est celui d'une poutre soumise à une flexion 03 points (Benchmark MECA), et le deuxième est celui d'une poutre soumise à une flexion cyclique. A la lumière de ces exemples nous avons constaté une très bonne cohérence et concordance entre les résultats de nos simulations et l'expérimentation d'une part et ceux d'autres modèles numériques de la littérature d'autre part. Nous avons constaté aussi, si le matériau est déchargé après avoir subi un état d'endommagement, il restaure sa raideur, les fissures précédemment ouvertes se referment mais la structure interne du matériau reste toujours endommagée.

\section{References}

1. P. Kotronis, Thèse de Doctorat, (Ecole normale supérieure de Cachan, 2000)

2. P. Kotronis, F. Ragueneau, J. Mazars, Engineering Structures, 27 (2005), P. 1197-1208

3. F. Ragueneau, Mémoire d'habilitation à diriger des recherches, (Université Pierre et Marie Curie, Paris 6, 2006)

4. M. Khebizi, H. Guenfoud, M. Guenfoud, Courrier du Savoir, 18, (2014), P. 111-115

5. M. Khebizi, M. Guenfoud, Computers and Concrete, 15, 4 (2015), P. 547-562

6. M. Khebizi, H. Guenfoud, M. Guenfoud, Challenge Journal of Structural Mechanics, 3, 1 (2017) P.52-57

7. C. Laborderie, Thèse de doctorat, (Université Paris 6, 1991).

8. C. Laborderie, Thèse d'habilitation à diriger les recherches, (Université de Pau et des Pays de l'Adour, 2003)

9. S. Ghavamian, MECA project benchmark, (EDP EDF R\&D, 2001)

10. M. Matallah, C. Laborderie, Engineering Fracture Mechanics, 76 (2009), P. 1087-1108. 